\title{
Clinical Outcomes in pT4 Tongue Carcinoma are Worse Than in pT3 Disease: How Extrinsic Muscle Invasion Should be Considered?
}

\author{
Carol M. Lewis, MD, MPH, FACS \\ Department of Head and Neck Surgery, University of Texas MD Anderson Cancer Center, Houston, TX
}

Through the consensus of task forces designated according to disease anatomic site, the American Joint Committee on Cancer (AJCC) Staging Manual has codified cancer staging since 1977; the Head and Neck Section is composed of 28 head and neck cancer experts. ${ }^{1}$ Staging systems aim to create guidelines that group patients with similar survival into subgroups (hazard consistency) that are distinct from other subgroups in terms of survival (hazard discrimination) but not cohort size (balance between groups) and that provide prognostic survival information on an individual patient level (high predictive ability). ${ }^{2}$

The recently released eighth edition of the AJCC Staging Manual, which will go into effect in 2018, made significant changes to the staging of oral cavity cancer. Most important was the incorporation of depth of invasion (DOI) into tumor (T) category determination. ${ }^{1}$ In the eighth edition, both tumor size and DOI determine categorization for $\mathrm{T} 1$ to $\mathrm{T} 3$, with the $\mathrm{T} 4$ category defined by invasion of local anatomic boundaries. However, as a result of the inclusion of DOI, extrinsic muscle invasion (EMI) was excluded as a criterion for the T4 category; the task force reasoned that limited EMI was difficult to definitively assess and can be supplanted by DOI. ${ }^{1}$ Interestingly, EMI was still recognized as a defining criteria for $\mathrm{T} 4$ oropharyngeal cancer, indicating that the task force recognized its importance in indicating an advanced cancer.

In this edition of Annals of Surgical Oncology, Liao and colleagues $^{3}$ at Chang Gung University in Taiwan challenge

\footnotetext{
(C) Society of Surgical Oncology 2017
}

First Received: 18 May 2017;

Published Online: 22 June 2017

C. M. Lewis, MD, MPH, FACS

e-mail: cmlewis@mdanderson.org the exclusion of EMI from oral cavity T4 category criteria. In a retrospective review of 203 consecutive patients with index oral tongue pT3 or pT4 squamous cell carcinomas (SCC), who were staged using the AJCC seventh edition (2010) criteria, this group identified significant differences in 5-year locoregional control (LRC) and disease-free survival (DFS), as well as the development of distant metastases (DM) between the pT3 and pT4 cohorts.

Liao et al. identified 135 pT3 and 68 pT4 consecutive patients with oral tongue SCC treated between 1996 and 2014; despite a long study period, patients were all staged by the AJCC seventh edition staging criteria. Patients underwent surgery with postoperative adjuvant therapy being determined by pathologic adverse features. Median follow up was 56 months for pT3 patients and 29 months for pT4 patients. Main outcome measures were 5-year LRC, DFS, and DM.

Interestingly, $93 \%$ of pT4 patients were staged as such due to EMI alone; these patients would be down-staged to pT3 under the current AJCC staging system. In addition, the authors identified DOI $>15 \mathrm{~mm}$ and DOI $>20 \mathrm{~mm}$ as significantly associated with the pT4 category, but the new staging system only recognizes DOI $>10 \mathrm{~mm}$ as an upstaging characteristic. Lastly, 4 (5.9\%) patients had EMI despite a DOI of $\leq 10 \mathrm{~mm}$ and tumor size $\leq 40 \mathrm{~mm}$, which would classify them as having pT2 tumors by the current AJCC staging system.

In comparing patients with pT3 and pT4 oral tongue SCC, Liao et al. found statistically significant differences in LRC (75 vs. 50\%, respectively; $p<0.001$ ), DFS (69 vs. $43 \%$, respectively, $p<0.001$ ), and DM (14 vs. $27 \%$, respectively, $p=0.013$ ). In multivariate analysis, the $\mathrm{pT} 4$ category was identified as an independent prognostic factor for LRC and DFS, but not overall survival (OS), perhaps confounded by the correlation of the pT4 category with other risk factors for death. 
This study was not without limitations. This was a retrospective review of patients during an 18-year period at a single institution. Only patients with oral tongue cancer were included, and all were treated with upfront surgery, so the generalizability of these findings with regard to other oral cavity subsites or for patients treated with other approaches is unclear. Additionally, although $93 \%$ of pT4 tumors had EMI alone, this was not examined as an independent prognostic factor; evaluating this specific covariate might have shed more light on EMI as an adverse feature of the $\mathrm{T} 4$ category.

AJCC staging systems evolve to incorporate new findings and data to improve the prognostic relevance of cancer staging; challenges to changes in the system are not uncommon. After AJCC published a unified TNM staging system with the International Union Against Cancer in 1988, some authors did not recognize a change in the T4 category, whereas others rallied to reincorporate a size delineation and pterygoid muscle invasion into the definition of T4 oral cavity cancer. ${ }^{4}$ Virag et al. reported significant differences in OS and DFS depending on whether the same cohort was staged with the previous or the new criteria. ${ }^{5}$ Indeed, Liao and colleagues have previously challenged the seventh edition of AJCC staging for T4 oral cavity cancers, reporting that despite being classified as $\mathrm{T} 4 \mathrm{~b}$ tumors based on masseteric space or pterygoid plate invasion, those below the mandibular notch had comparable LRC, DM, and DFS as T4a tumors. ${ }^{6}$ These data suggest that $\mathrm{T} 4 \mathrm{~b}$ category criteria should be reexamined, although they remain unchanged in the eighth edition.

The incorporation of DOI into the most recent staging system is significant and is overdue. The importance of DOI was recognized at least as far back as the 1980s, with several groups formulating new staging criteria that would add DOI to existing AJCC staging, or based solely on diameter and DOI without anatomic criteria. ${ }^{7-9}$ Relevant to this discussion of EMI are past studies recommending that EMI be excluded from the T4 classification. ${ }^{10,11}$ In an anatomic study, Boland et al. found that the extrinsic tongue muscles exist a few millimeters below the mucosa, especially laterally, such that involvement does not necessarily reflect deep invasion or aggressiveness. ${ }^{10}$ This may explain the four patients in the present study for whom DOI was $<10 \mathrm{~mm}$, although EMI was present on histopathology. Additionally, Boland et al. created a computer model to review archived pretreatment magnetic resonance imaging (MRI) studies of 87 oral tongue cancer patients, finding that $52 \%$ of patients would be up-staged to the T4a category based on this criteria. Further analysis revealed that although EMI significantly affected OS and DFS, it did not impact disease-related survival. ${ }^{11}$ These authors concluded that EMI should not be a criterion for the T4 category. In contrast, Liao et al identified EMI pathologically, which is arguably a more definitive assessment of EMI.

Whether EMI is an independent prognostic factor in oral tongue cancer remains unclear; even in this paper by Liao et al., EMI was not examined independent of T category. In light of previous publications regarding DOI and EMI, the AJCC Head and Neck Section's rationale for excluding EMI, based upon uncertain assessment in cases of mild EMI and because newly incorporated DOI criteria should supplant EMI, is reasonable. However, AJCC staging criteria continually evolve to reflect current evidence offering more precise predictors of prognosis. Liao and colleagues should be commended for challenging the supposition that DOI can supersede EMI in oral cavity T4 category criteria by reporting data suggesting that it does not. Further study will be needed to determine more definitively the significance of EMI in the prognosis of oral tongue cancer.

\section{REFERENCES}

1. Lydiatt WM, Patel SG, O'Sullivan B, et al. Head and neck cancers-major changes in the American Joint Committee on cancer eighth edition cancer staging manual. CA Cancer J Clin. 2017;67(2):122-37.

2. Groome PA, Schulze K, Boysen M, Hall SF, Mackillop WJ. A comparison of published head and neck stage groupings in carcinomas of the oral cavity. Head Neck. 2001;23(8):613-24.

3. Liao C-T, Lee L-Y, Hsueh C, et al. Clinical outcomes in pT4 tongue carcinoma are worse than in pT3 disease: how extrinsic muscle invasion should be considered? Ann Surg Oncol. 2017. doi:10.1245/s10434-017-5906-3.

4. Ghouri AF, Zamora RL, Harvey JE, Spitznagel EL Jr, Sessions DG. Epidermoid carcinoma of the oral cavity and oropharynx: validity of the current AJCC staging system and new statistical tools for the prediction of subclinical neck disease. Otolaryngol Head Neck Surg. 1993;108(3):225-32.

5. Virag M, Uglesic V, Aljinovic N, Jurlina M. Are the new staging rules better for T4 oral cancer? Am J Surg. 1995;170(5):432-5.

6. Liao CT, Lee LY, Hsueh C, et al. Comparative outcomes in oral cavity cancer with resected pT4a and pT4b. Oral Oncol. 2013;49(3):230-6.

7. Moore C, Kuhns JG, Greenberg RA. Thickness as prognostic aid in upper aerodigestive tract cancer. Arch Surg. 1986;121(12):1410-4.

8. International Consortium for Outcome Research in Hand and Neck Cancer, Ebrahimi A, Gil Z, et al. Primary tumor staging for oral cancer and a proposed modification incorporating depth of invasion: an international multicenter retrospective study. JAMA Otolaryngol Head Neck Surg. 2014;140(12):1138-48.

9. Howaldt HP, Kainz M, Euler B, Vorast H. Proposal for modification of the TNM staging classification for cancer of the oral cavity. DOSAK. J Craniomaxillofac Surg. 1999;27(5):275-88.

10. Boland PW, Pataridis K, Eley KA, Golding SJ, Watt-Smith SR. A detailed anatomical assessment of the lateral tongue extrinsic musculature, and proximity to the tongue mucosal surface. Does this confirm the current TNM T4a muscular subclassification? Surg Radiol Anat. 2013;35(7):559-64.

11. Boland PW, Pataridis K, Eley KA, Golding SJ, Watt-Smith SR. Automatic upstaging of tongue squamous cell carcinoma with lateral extrinsic muscle involvement is not justified. Int J Oral Maxillofac Surg. 2013;42(11):1397-402. 